EPJ Web of Conferences 71, 00057 (2014)

DOI: 10.1051/epjconf/20147100057

(C) Owned by the authors, published by EDP Sciences, 2014

\title{
ALICE Masterclass on strangeness
}

\author{
Panagiota Foka ${ }^{1, a}$ and Małgorzata Janik ${ }^{2, b}$ \\ ${ }^{1}$ GSI Helmholtzzentrum fur Schwerionenforschung GmbH, Planckstrasse 1, 64291 Darmstatd, Germany \\ ${ }^{2}$ Faculty of Physics, Warsaw University of Technology, ul. Koszykowa 75, 00-662 Warszawa, Poland
}

\begin{abstract}
An educational activity, the International Particle Physics Masterclasses, was developed by the International Particle Physics Outreach Group with the aim to bring the excitement of cutting-edge particle-physics research into the classroom. Thousands of pupils, every year since 2005, in many countries all over the world, are hosted in research centers or universities close to their schools and become "scientists for a day" as they are introduced to the mysteries of particle physics. The program of a typical day includes lectures that give insight to topics and methods of fundamental research followed by a "hands-on" session where the high-school students perform themselves measurements on real data from particle-physics experiments. The last three years data from the ALICE experiment at LHC were used. The performed measurement "strangeness enhancement" and the employed methodology are presented.
\end{abstract}

\section{Introduction}

The International Particle Physics Outreach Group (IPPOG) [1] is a network of scientists, researchers, science educators and communication specialists engaged in informal science education and outreach for particle physics. Within the framework of IPPOG an educational project, called International Masterclasses (IMC) [2], was developed with the aim to bring experimental data and research methods in the classroom. The main idea was to give high-school students, aged 15-18, the possibility to experience how scientists investigate the laws of nature by doing it themselves. The students and their teachers are invited to a university or research center near their school for a day. There, students and teachers get acquainted with topics and methods of basic research and on the fundamental laws of matter and forces. The highlight of the day and the main idea of the Masterclass is to give them the possibility to perform measurements on real data from particle-physics experiments, as real scientists do.

The experiments at the Large Hadron Collider (LHC) at the European Organization for Nuclear Research (CERN) collected a large amount of data. Very soon interesting new results were produced and published by all LHC collaborations; the highlights being the discovery of the Higgs and the award of the 2013 Nobel Price for Physics, just four years after the LHC startup. Naturally, the wish is to share this excitement with the general public and in particular with school children, the potentially future generations of scientists. Hence, already in 2010 the International Masterclasses

\footnotetext{
a e-mail: yiota.foka@cern.ch

be-mail: majanik@if.pw.edu.pl
}

This is an Open Access article distributed under the terms of the Creative Commons Attribution License 2.0, which permits unrestricted use, distribution, and reproduction in any medium, provided the original work is properly cited. 
featured a sample of the first LHC data from the ALICE, ATLAS and CMS experiments as detailed in [3-5]. These were soon updated, as the analysis progressed, containing a sample of Higgs events. Educational material, methods and analysis tools have been developed to accommodate the new data. The emphasis varies from sharing the enthusiasm of the field to explaining to the teachers and young students how things really work through active participation and involvement in "do-it-yourself" measurements and searches.

A centrally organized event runs every year during the month of March. Institutes and universities all over the world host for a day a class of high-school students. A maximum of five institutes participate during the same day doing the same measurement and at the end of the day they join a videoconference to present and discuss their results. Depending on the time zone, the video-conference is coordinated and moderated either by CERN or Fermilab.

The Masterclass packages, however, are widely used for many other occasions, including events at different schools.

\section{ALICE Masterclass}

\subsection{ALICE Experiment}

ALICE, A Large Ion Collider Experiment [6], is the heavy-ion dedicated experiment at LHC. Its main goal is to search for and characterize the properties of a deconfined state of quarks and gluons, called quark-gluon plasma (QGP). According to the theory, this state of matter existed in the early universe and exists in the cores of neutron stars. Such a state of matter is also expected to be produced at the laboratory, above certain critical conditions of temperature and energy density, which are reached and exceeded in collisions of lead ions at the LHC. Contrary to the discovery of a new particle, one has to investigate several observables to draw conclusions about the creation of the QGP and its properties. In addition, one has to compare such observables to results from elementary proton-proton collisions, which are used as a baseline measurement. One of the first predicted signatures of the QGP was enhanced production of "strange" particles relative to the one in $p p$ collisions.

Selected highlights of ALICE experimental results, mainly from the analysis of $\mathrm{Pb}-\mathrm{Pb}$ collisions at $\sqrt{s_{\mathrm{NN}}}=2.76 \mathrm{TeV}$, can be found in [7-9].

\subsection{Strangeness Enhancement}

Strangeness is one of the "historic" observables in ultrarelativistic nucleus-nucleus collisions [1012]. In elementary collisions, strangeness production is suppressed relative to the production of light flavors. For a large system, this suppression is expected to be removed - i.e. strangeness production is expected to be enhanced - provided the system allows for a thermodynamical description in which strangeness conservation can be treated over a large volume and does not need to be enforced locally at each collision, or, in other words, provided the correlation volume is also large, as expected if partons are freed in a quark-gluon plasma $[13,14]$. This is non-trivial: if, for instance, nucleus-nucleus collisions were just a simple superposition of independent nucleon-nucleon collisions, no strangeness enhancement would be expected, no matter how large the system size. The crucial difference is that macroscopic bulk matter ensures communication within the system through long-range correlations.

A review on some aspects of the role of strange quarks in hot QCD matter and as probes of quark deconfinement at high temperature was presented by B. Muller in SQM 2011 [15] and is briefly summarised below. Strange quarks play a crucial role in shaping the phase diagram of QCD matter. They are also excellent probes of the structure of QCD matter, because: 
1. they are hard to produce at temperatures below the critical temperature, $T_{c}$, since their effective mass is much larger than $T_{c}$ when chiral symmetry is broken, but easy to produce at temperatures above $T_{c}$ since the current mass of the strange quark $m_{s} \approx 100 \mathrm{MeV}<T_{c}$;

2. quark flavor is conserved under the strong interactions implying that strange quarks, once produced, are not easy to destroy during the dilute hadronic freeze-out stage of heavy-ion reactions.

The original idea of enhanced production of hadrons containing strange quarks as a signature of quark deconfinement, proposed by Rafelski and Hagedorn in 1980 [16], was based on the insight that strange antiquarks are at least as abundant as light antiquarks in quark matter for $\mu_{B}>0$, and can be substantially more abundant, e.g. when $\mu_{B} \sim T$ in the domain of collision energies accessible at the CERN-SPS.

Soon after, Rafelski also discussed the role of multi-strange antibaryons $\bar{\Xi}, \bar{\Omega}$ and the $\phi$-meson, as probes of the QGP [17] explaining that the formation of a baryon-rich, flavor equilibrated quark-gluon plasma would result in their copious production. ${ }^{1}$

The additional argument, that the flavor composition of a transient quark-gluon plasma could be equilibrated during the short period of its existence, was supplied in 1982 by the calculation [14] of the rate of strange quark pair production in a deconfined QCD plasma. The crucial observation was that the process $g g \rightarrow s \bar{s}$ is the dominant production channel. Thus an enhanced production of hadrons containing multiple strange quarks in nucleus-nucleus collisions, compared with $p p$ collisions, not only signals quark deconfinement, but also the liberation of gluons as dynamical excitation modes in hot QCD matter. In other words, strangeness enhancement, as the effect was called in brief, provides a signal for the formation of a quark-gluon plasma in the true sense of the term.

The history of this insight was described in great detail by Rafelski in the Proceedings of the Zimanyi Memorial Conference [18]. The full set of experimental implications of enhanced strange quark production were published in a Physics Reports article by Koch et al. [19], including the following main prediction. As a consequence of quark-gluon plasma formation in relativistic heavyion collisions, all strange hadrons, including multi-strange baryons and antibaryons, will be produced in relative chemical equilibrium abundances. This is a natural consequence of hadron formation by statistical coalescence of deconfined quarks.

While this prediction seemed preposterous at the time, it is a fair statement that of all the quarkgluon plasma signatures proposed in the 1980's, equilibrated strangeness production has been quantitatively confirmed in every detail by the experimental data. The predicted enhancement of strange (anti-)baryon production has been observed in collisions of heavy nuclei ( $\mathrm{Pb}-\mathrm{Pb}$ or $\mathrm{Au}-\mathrm{Au}$ ) at $\mathrm{SPS}$, RHIC, and most recently, LHC, as presented in SQM 2011 conference.

In order to make quantitative predictions for the expected hadron abundances in the final state of a relativistic heavy-ion reaction it is important to understand how the quark-gluon plasma hadronizes. Rafelski and Danos addressed this issue in 1987 [20]. They distinguished between fragmentation and recombination mechanisms and pointed out that recombination is expected to be the dominant mechanism of hadron emission at energies above the mean thermal energy. In this domain, the enhanced strangeness production would most directly reflect the strange quark abundance and quark deconfinement in the quark-gluon plasma phase.

In general, extensive analysis of experimental data show that bulk particle production can be very well described in the framework of the thermal (statistical) hadronisation model with a small set of adjustable parameters [21]. The most important ones are the chemical temperature $T_{\mathrm{ch}}$, the baryon chemical potential $\mu_{B}$, and the strange phase space saturation factor $\gamma_{s}$.

\footnotetext{
${ }^{1}$ This prediction was greeted almost with disbelief by many physicists in view of the fact that these particles are extremely rarely produced in $p p$ collisions at SPS.
} 
The thermal (statistical) model assumes that particles are created in thermal (phase space) equilibrium governed by a scale parameter $\mathrm{T}$, interpreted as a temperature. The production of a particle with mass $m$ is suppressed by a Boltzmann factor $e^{-m / T}$. Conservation laws introduce additional constraints, like the baryochemical potential $\mu_{B}$ which accounts for baryon number conservation. The chemical potential of the strange quark, $\mu_{s}$, is fixed by the requirement that the net strangeness of the matter must vanish. In the canonical formalism, applied to a finite matter volume, the fugacity factor $\gamma_{s}$ receives a contribution from strangeness conservation. Thus $\gamma_{s}$ is predicted to be less than unity in a finite volume even at full chemical equilibrium [11]. However, $\gamma_{s}$ can exceed unity at hadronization, if strange quarks are chemically equilibrated at $T \gg T_{c}$ and the annihilation reactions cannot keep up with the cooling rate of the expanding quark-gluon plasma [22]. In reality, this apparent dichotomy may be an artificial consequence of our ignorance of the nature of the structure of strongly interacting matter near $T_{c}$. Since the transition between hadronic matter and quark-gluon plasma is not a discontinuous phase transition but a cross-over, it is possible that QCD matter in the transition regime may be equally well described as a dense gas of Hagedorn resonances or as a strongly coupled plasma of quarks and gluons.

This additional parameter $\gamma_{s}$ describes the observation that in some collision systems particles containing strange quarks are suppressed compared to the grand canonical thermal expectation. The temperature parameter is found in all high energy collisions $\left(p p, e^{+} e^{-}, \mathrm{A}+\mathrm{A}\right)$ to be about $160-170$ $\mathrm{MeV}$, while $\gamma_{s}$ increases from $0.5-0.6$ in $p p$ to $0.9-1$ in $\mathrm{A}+\mathrm{A}[12,23]$. This disappearance of strangeness suppression in nuclear collisions [24, 25], the so-called strangeness enhancement, and the fact that the bulk of all particles are produced in heavy-ion reactions with thermal ratios to very good approximation (typically $<10-20 \%$ ) is considered to be an essential and well established fact.

The LHC data have shown that particles and anti-particles are produced in $\mathrm{Pb}-\mathrm{Pb}$ collisions at midrapidity in equal numbers, consistent with the very small value of the baryochemical potential $\mu_{B}$ of the thermal model. Strangeness is enhanced in $\mathrm{Pb}-\mathrm{Pb}$ compared to $p p$, by a factor which increases with strangeness content from about 1.3 in $\mathrm{K} / \pi$ to up to $>3$ in $\Omega / \pi$. All strange particle ratios in $\mathrm{Pb}-$ $\mathrm{Pb}$ are very well described by the thermal model, implying that they are produced in accordance with fully thermal ratios (i.e. $\gamma_{S}=1$ ). However, in a complete surprise, protons were found to be lower than the thermal model expectations (by a factor of about 1.5) and well outside the usual precision of the thermal model. Continuous investigations aim to clarify the source(s) and the significance of the observed effect. Several possible explanations for these deviations have been suggested. In particular large baryon-antibaryon annihilation rates in the late hadronic phase could be the source of some lower baryon yields.

In summary experiments at the SPS, RHIC, and the LHC have shown that the phase space of strangeness is fully equilibrated in ultrarelativistic heavy-ion collisions, as was theoretically predicted 30 years ago. Formation of deconfined QCD matter, the quark-gluon plasma, is the most natural mechanism for strangeness equilibration.

However, a quantitative understanding of the experimental data raises many questions about the quark-gluon plasma-to-hadron gas transition. Detailed analysis of the data coming from RHIC and LHC together with improved theoretical studies will contribute to further investigate the nature of this transition as well as the structure of the QCD phase diagram.

\subsection{Tools and didactic advantages}

The ALICE Masterclass package relies on the measurement of strange particles in $\mathrm{Pb}-\mathrm{Pb}$ collisions and their comparison to results from $p p$ collisions.

ALICE event display is based on AliRoot [26], the ALICE experiment dedicated software based upon ROOT [27], framework designed for the analysis of data coming from high energy physics 
experiment. While the ALICE event display can be used, a simplified version of it has been developed which is independent on heavy software of ALICE and is based on ROOT only. However, it was designed to be very similar to the standard ALICE event display environment that is used in the ALICE Control Room (for comparison see Fig. 1).
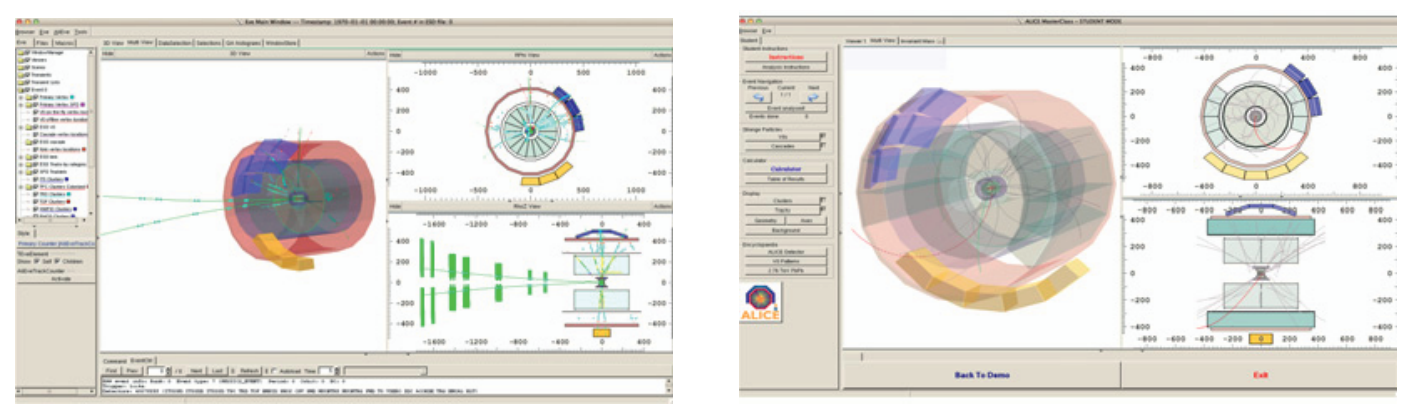

Figure 1. ALICE event display used in the ALICE control room (left), adaptation of ALICE event display for the Masterclass (right).

The Masterclass Event Display inherits powerful properties that the ALICE Event Display possess, and allows, in addition to the interactive 3D visual inspection of the events, different other functions like filling of histograms and tables within the same environment [28].

In the 2013 version of the ALICE Masterclass the students were first given small $p p$ and $\mathrm{Pb}-\mathrm{Pb}$ data samples for such visual inspection. By manipulating interactively the events in $3 \mathrm{D}$ they better appreciate the differences between $p p$ and $\mathrm{Pb}-\mathrm{Pb}$ events of much higher multiplicity and are much more complex. They also get acquainted with the concepts of tracking and particle identification, learning that particles bend in a magnetic field and the kind of information one can extract from this. Strange particles decay after flying for some distance in the detectors leaving a characteristic decay pattern, known as V0 or cascade, which the students learn how to identify.

In addition to the visual inspection of individual events, the Masterclass package also implements methods to analyze large statistics samples of $p p$ and $\mathrm{Pb}-\mathrm{Pb}$ collisions. A fitting procedure is implemented that allows students to choose the limits for the fit, fit separately the background and the peak of the signal and subtract them to obtain the number of strange particles. The results obtained from the analysis are finally compared to published results (see Fig. 2).

\subsection{History and prospects}

Strangeness enhancement Masterclass measurement has in fact followed the evolution of the real life experiment and is updated and enriched every year. On the first year there was only $p p$ data available. Students analyzed proton-proton collisions to establish the reference and also investigate if high multiplicity $p p$ at LHC is similar to heavy-ions. Such studies are also very important to fine-tune the Monte Carlo models.

When $\mathrm{Pb}-\mathrm{Pb}$ data came it was possible for students to analyze also heavy-ion collisions. By comparing $p p$ with $\mathrm{Pb}-\mathrm{Pb}$ the strangeness enhancement factor could be calculated and measured for different centrality classes.

However, there is still place for further developments. The studies of hadron yields in a framework of statistical / thermal models would allow to extract the hadronisation temperature. 

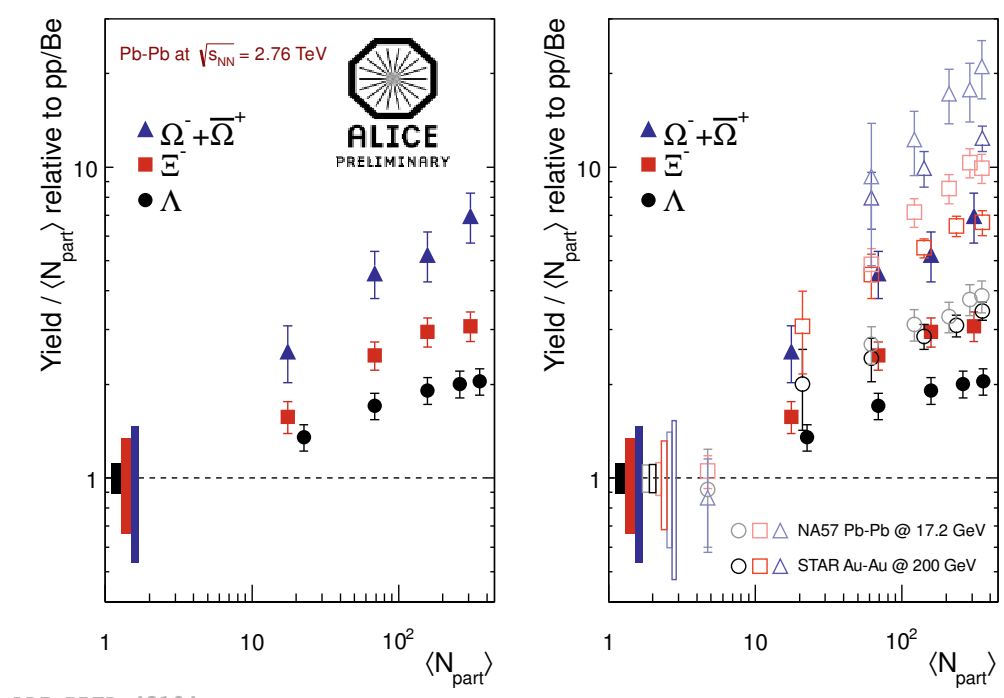

Figure 2. Strangeness enhancement for $\Lambda, \Xi^{-}$and $\Omega^{-}+\Omega^{+}$baryons in ALICE (left plot) and compared to other experiments (right plot).

\subsection{Didactic process}

It is very important for students to understand the entire "flow" of the measurement: the theoretical concepts, limitations of the analysis "by eye", strengths of merging the results and working in collaboration and the conclusions about QGP. This is the reason that the proposed measurement has a certain structure and consist of several steps.

\subsubsection{Theoretical introduction}

In a simplified way we use the argument that we start with two nuclei which contain protons and neutrons made out of up and down quarks. However, after the collision we detect in our aparatus "strange particles" containing strange quarks. Where did they come from? It is reasonable to assume that they come from a possible QGP state created during the collision (but we have to be careful to explain that any other possible scenarios should be studied and excluded). After the theoretical introduction to the whole concept the students can sit in front of the computers and verify the story themselves.

\subsubsection{Visual analysis}

At first the students and tutors, together analyze examples of decays of strange particles: $K, \Lambda, \bar{\Lambda}, \Xi^{-}$. The visual analysis of the events facilitates the explanation of "how to see the strange particles" and also allows to easily communicate basic concepts (i.e. the behavior of charged particles in magnetic field, particle identification techniques etc.). Moreover, it can be pointed out that a very similar event display is used online in the ALICE Control Room to monitor the quality of recorded collision data, as well as to verify and tune algorithms and methods. 
After the common exercise is performed by the whole class, students get to work by themselves at the computers, in groups of 2 or 3; they look at 15 events, find appropriate patterns, calculate the masses of initial particles from the momentum components of the daughter particles using invariant mass calculator, classify them as one of the mentioned strange particles, update tables and histograms. When the work is completed the tutor collects the results from each group and merges them. After collecting the results from the whole class it becomes clear that still only a small fraction of the data was analyzed. One needs statistics, and obtaining it by analysing manually events one-by-one would take a lot of time. Students are also shown the $\mathrm{Pb}-\mathrm{Pb}$ sample and it is obvious that scanning such events on the Event Display has limitations for real analysis; too many tracks make it impossible to find the ones we are looking for.

\subsubsection{Large scale analysis}

During the large statistics analysis students analyze 20000 events by using build-in algorithms activated through the event display panel, looking for $K, \Lambda$ and $\bar{\Lambda}$. In the visual analysis there was no background present, since visually students selected the correct tracks. However when strange particle decay patterns are found by the algorithm, the misidentification rate is much higher. It is explained to the students how to fit the peak and the background to extract the number of strange particles in the peak. Students find the number of $K, \Lambda$ and $\bar{\Lambda}$ in a large sample of $p p$ events and then in a large sample of $\mathrm{Pb}-\mathrm{Pb}$ events. After this short tutorial they work by themselves at computers; each group is assigned a centrality region and has to calculate the number of strange particles in this sample. Figure 3 shows a snapshot of the "Large Scale" analysis tool, which contains V0 and cascade decay finders, and allows the calculation of invariant masses filling of histograms as well as fitting the distributions.

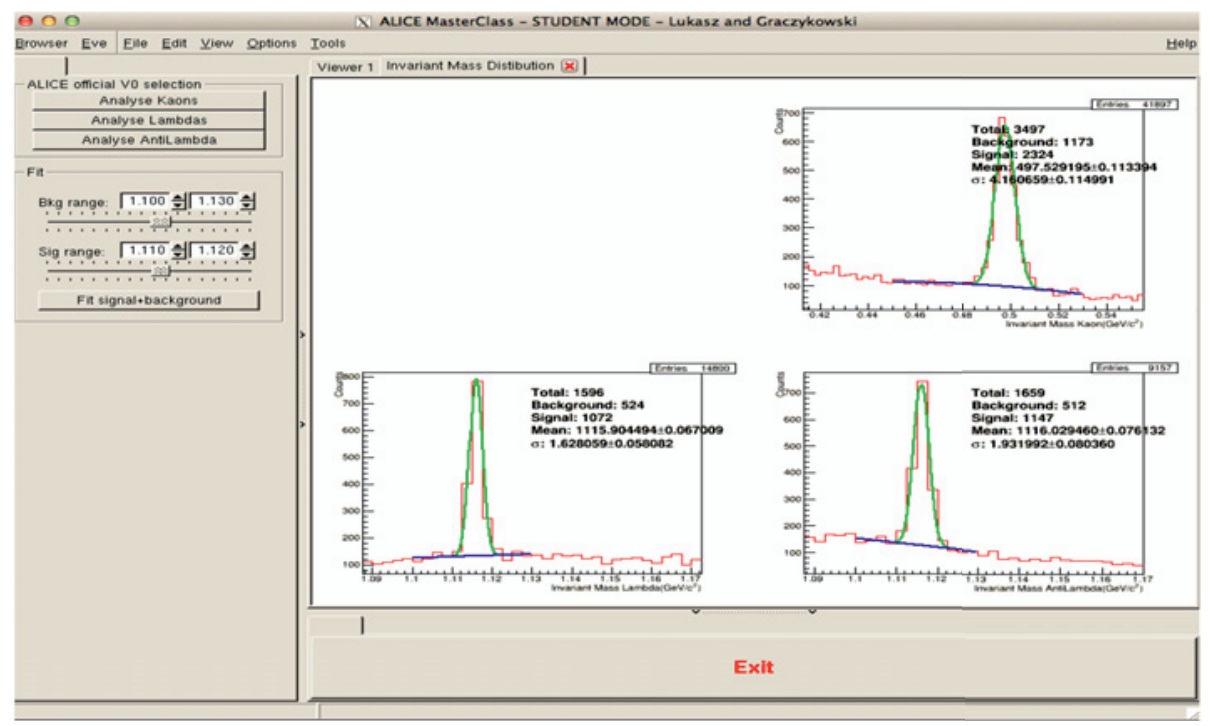

Figure 3. Large Scale Analysis tool, with invariant mass plots for $\mathrm{K}, \Lambda$ and $\bar{\Lambda}$.

The exercise ends when students collect the results in online spreadsheet. They are given new set of data: mean number of participatig nucleons in the event (based on analyzed centrality), number of analyzed events and efficiency of the detector. First students have to calculate the particle yield, and 
then strangeness enhancement: the yield normalized by the number of participating nucleons in the $\mathrm{Pb}-\mathrm{Pb}$ collision, and divided by the observed yield in proton-proton collisions. The tutor discusses the obtained results and their relevance to the physics questions exposed in the morning. The students prepare the final result of the classroom and a small presentation.

\section{Conclusions}

The aim of the ALICE 'strangeness enhancement' measurement is to identify strange particles and give pupils a flavor of analyzing the data with real analysis tools. It allows to explain basic concepts in high energy particle physics and at the same time make students feel like "the real scientist".

\section{References}

[1] International Particle Physics Outreach Group - IPPOG, http://ippog.web.cern.ch, accessed: 2013-12-20

[2] International Masterclasses - hands on particle physics, http://www.physicsmasterclasses.org/, accessed: 2014-02-11

[3] F. Ould-Saada, PoS ICHEP2012, 559 (2013)

[4] P. Foka, PoS ConfinementX, 029 (2012)

[5] P. Foka, to be published in ICHEP Proceedings ICFP 2012 (2014)

[6] K. Aamodt et al. (ALICE Collaboration), JINST 3, S08002 (2008)

[7] K. Safarik (ALICE Collaboration), Nucl.Phys. A904-905, 27c (2013)

[8] P. Foka (the ALICE Collaboration), J.Phys.Conf.Ser. 455, 012004 (2013)

[9] M. Janik, Proc. of SPIE 8903 (2013)

[10] F. Antinori, J.Phys. G35, 044055 (2008)

[11] S. Hamieh, K. Redlich, A. Tounsi, Phys.Lett. B486, 61 (2000)

[12] A. Andronic, P. Braun-Munzinger, J. Stachel, Acta Phys.Polon. B40, 1005 (2009)

[13] J. Rafelski, M. Danos, Phys.Lett. B97, 279 (1980)

[14] J. Rafelski, B. Muller, Phys.Rev.Lett. 48, 1066 (1982)

[15] B. Muller, Acta Phys.Polon. B43, 761 (2012)

[16] J. Rafelski, R. Hagedorn, CERN-TH-2969, C80-08-24-4 (1980)

[17] J. Rafelski, Phys.Rept. 88, 331 (1982)

[18] J. Rafelski, Eur.Phys.J.ST 155, 139 (2008)

[19] P. Koch, B. Muller, J. Rafelski, Phys.Rept. 142, 167 (1986)

[20] J. Rafelski, M. Danos, Phys.Lett. B192, 432 (1987)

[21] B. Muller, J. Schukraft, B. Wyslouch, Ann.Rev.Nucl.Part.Sci. 62, 361 (2012)

[22] J. Letessier, J. Rafelski, Phys.Rev. C75, 014905 (2007)

[23] F. Becattini, P. Castorina, A. Milov, H. Satz, Eur.Phys.J. C66, 377 (2010)

[24] C. Blume, C. Markert, Prog.Part.Nucl.Phys. 66, 834 (2011)

[25] S. Margetis, K. Safarik, O. Villalobos Baillie, Ann.Rev.Nucl.Part.Sci. 50, 299 (2000)

[26] AliRoot Documentation, http://www.physicsmasterclasses.org/, accessed: 2014-02-26

[27] ROOT, http://root.cern.ch/, accessed: 2014-02-07

[28] AliEve, http://aliweb.cern.ch/Offline/Activities/Visualisation/index.html, accessed: 2014-02-07 